\title{
THE DESIGN OF MEDIUM PRF RADAR SCHEDULES FOR OPTIMUM DETECTABILITY IN DIVERSE CLUTTER SCENES
}

\author{
C.M. Alabaster, E.J. Hughes \\ Cranfield University, DCMT, Shrivenham, OXON SN6 8LA, UK \\ c.m.alabaster@cranfield.ac.uk \\ Fax: +44 (0)1793 785902
}

Keywords: Medium PRF, Evolutionary Algorithm, Clutter, Detectability.

\begin{abstract}
Airborne fire control radars using medium pulse repetition frequency (PRF) waveforms are required to maintain good target detectability in various clutter scenes. This paper describes work to optimise PRF values of 3 of 8 medium PRF schedules in varying clutter scenes in order to achieve optimal target detectability. To this end, the detectability map is introduced as a means of illustrating and quantifying target detectability over the full Range/Doppler space of interest. The paper concludes that optimised PRF sets achieve similar detectability performances irrespective of the clutter scene.
\end{abstract}

\section{Introduction}

Previous work [1-4] has sought to optimise the selection of precise values of pulse repetition frequency (PRF) for a medium PRF schedule of an airborne fire control radar (FCR). The optimisation process centred around an evolutionary algorithm which was driven so as to minimise range/Doppler blindness in the presence of homogenous clutter. This previous work employed simplistic models of the FCR antenna characteristics and the generation of clutter maps but was nevertheless successful in identifying several near-optimum PRF schedules. The current work utilises a similar evolutionary approach to select and trial PRF values but is now driven to maximise target detectability. Furthermore, a more sophisticated antenna and clutter model is now used which can model regions of differing clutter statistics. In this way it has been possible to explore the extent to which the design of the medium PRF waveform may be optimised for various clutter scenes, including inhomogeneous clutter representing land and sea. The metric used to assess the quality of each medium PRF schedule is the "detectability map", proposed by the authors here and explained later in this paper. Whilst the specific results obtained in this study pertain to the airborne FCR model used here it is worth noting that the general trends apply to other similar radars and also to airborne surveillance and early warning radars operating over both land and sea.

Section 2 of this paper describes the factors determining target detectability in medium PRF modes. This section describes the optimisation process (including the radar and clutter modelling) and introduces the idea of a detectability map to illustrate target detectability. Section 3 describes the evolutionary approach and the computer based experimental work. The results are presented in section 4 and finally, section 5 concludes.

\section{Target Detectability in Medium PRF}

\subsection{Medium PRF Schedules}

The selection of precise values of PRF in a medium PRF schedule are subject to the following constraints: decodability and the avoidance of ghost targets, minimal/ blindness and maximum detectability, avoidance of blind velocities and system limitations on maximum, minimum and mean PRF. Schedules are designed to yield target data in a minimum number of PRFs $(M)$ from a total number transmitted in the schedule $(N)$ in what is known as an $M$ of $N$ schedule. Typically, 3 of 8 schedules have been popular in airborne FCR applications but earlier work has indicated that 2 of 6 schedules are viable and even advantageous in some respects [2]. The optimisation process is illustrated in Figure 1. Parameters of the FCR are given in Table 1. The present work is based on 3 of 8 schedules as these are the most common. 


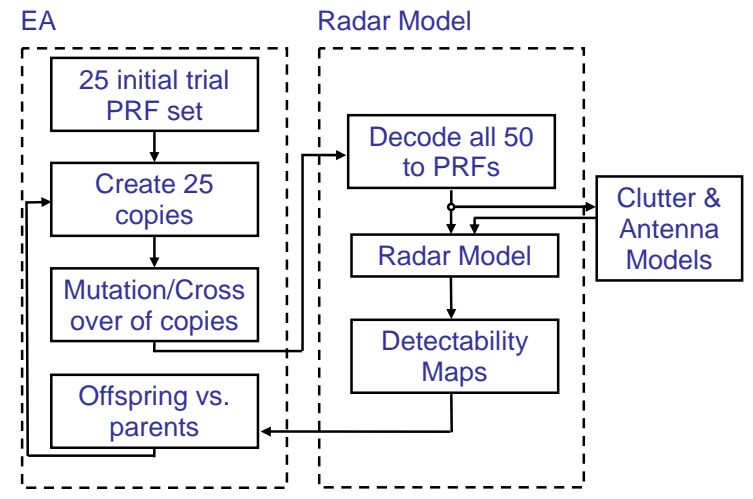

Figure 1: Optimisation Process

\begin{tabular}{|l|l|}
\hline Parameters & Value \\
\hline Carrier frequency & $10 \mathrm{GHz}$ \\
Max \& Min PRI & 150 to $35 \mu \mathrm{s}$ \\
PRI resolution & $100 \mathrm{~ns}(1151$ PRIs $)$ \\
Transmitted pulse width & $7 \mu \mathrm{s}$ \\
Compressed pulse width & $0.5 \mu \mathrm{s}$ \\
FFT size & 64 point \\
PRF changeover time & $1.7 \mathrm{~ms}$ \\
Blind range due to eclipsing & $15 \mathrm{range}$ cells \\
Duty cycle & Variable $(0.2$ peak $)$ \\
Beamwidth & $3.9^{0}$ \\
Scan rate & $60^{0} / \mathrm{s}$ \\
Target illumination time & $65 \mathrm{~ms}$ \\
Maximum target Doppler & $\pm 100 \mathrm{kHz}(1500 \mathrm{~m} / \mathrm{s})$ \\
Maximum detection range & $185.2 \mathrm{~km}(100 \mathrm{nmi})$ \\
Platform altitude & $5000 \mathrm{~m}$ \\
Platform velocity & $250 \mathrm{~m} / \mathrm{s}$ \\
Antenna depression angle & $6^{0}$ \\
\hline
\end{tabular}

Table 1: FCR Model

\subsection{Clutter Model}

The clutter model (which combines the FCR antenna model) generates the clutter radar cross section (RCS) as a function of grazing angle at every location on the ground within the radar range. The antenna is modelled as having a sinc squared (power) radiation diagram over a full sphere. The pattern may be offset in azimuth and elevation to account for beam steering angles with respect to the velocity vector of the platform. Platform motion compensation (PMC) is also applied to centre mainbeam clutter (MBC) to zero Doppler. Clutter has a Weibull distribution whose parameters may be adjusted for land or sea. Furthermore, the model imparts a random Doppler (Gaussian, mean $=0, \sigma_{\mathrm{n}}=5 \mathrm{~m} / \mathrm{s}$ ) onto the clutter to mimic slow ground moving targets (GMT). The clutter RCS is cascaded with the antenna gain and duly range and Doppler gated in accordance with the FCR model and PRF. A typical clutter map folded into one ambiguous range and Doppler interval is illustrated in Figure $2(\mathrm{PRF}=$ $10 \mathrm{kHz})$.

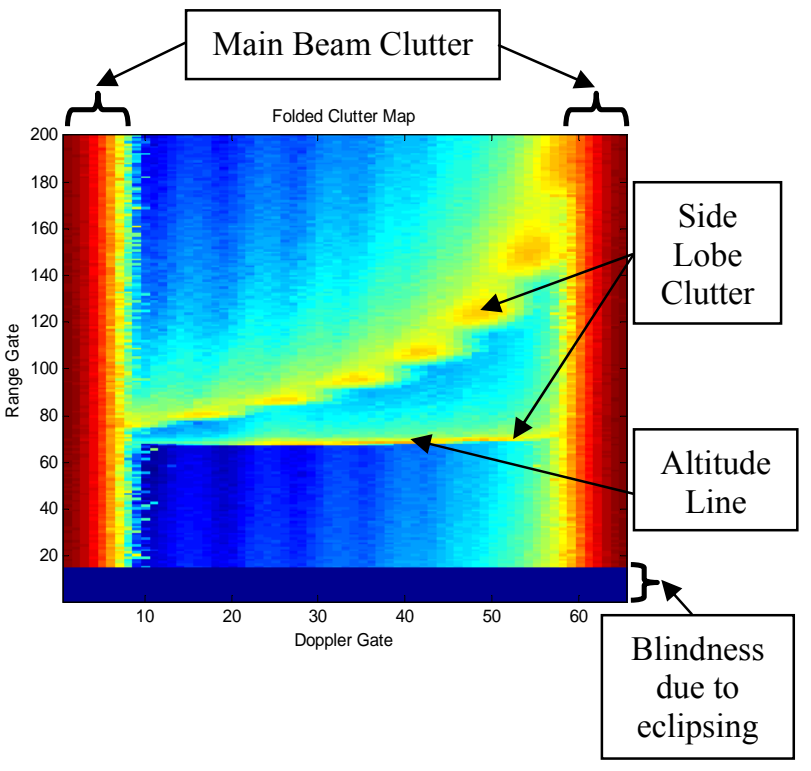

Figure 2: Folded Clutter Map

Figure 2 illustrates strong bands of MBC at zero Doppler and aliased at a Doppler equal to the PRF. These appear at the left and right hand edges, respectively. Sidelobe clutter (SLC) is spread across the entire Range/Doppler detection space. Of specific interest is the rather strong altitude line corresponding to the shortest range clutter directly underneath the platform.

Clutter was generated using a variable with a Weibull power distribution given in Equation (1).

$$
w=B \sqrt[c]{-\ln (U)}
$$

where $U$ is a uniformly distributed random number in the range $[0,1], B$ is the distribution spread and $C$ is the shape parameter. For land clutter $B=C=1$ whereas for sea clutter $B=0.1$ and $C=0.65$.

\subsection{Detectability Map}

The folded clutter map of Figure 2 is replicated in range and Doppler over the full detection space of interest (i.e. $185 \mathrm{~km}$ in range by $1500 \mathrm{~m} / \mathrm{s}$ in velocity) due to the repetition of data in the time and frequency domains. This results in an unfolded clutter map and is illustrated in Figure 3. Each PRF in the schedule has a similar, though different, unfolded clutter map. The probability of detection of a discrete target 
at any range/Doppler cell of interest depends on the number of PRFs in which the range/Doppler cell is not eclipsed and the probability of detection in each PRF, as determined by the signal to clutter ratio (SCR) of the cell. Blindness results from eclipsing, with no MBC/GMT blanking being assumed. A detectability map can therefore be derived over the full range and Doppler detection space of the radar and denotes the minimum target RCS required for detection at each range and Doppler cell in an appropriate number of PRFs. An example detectability map is given in Figure 4 based on a required $\mathrm{SCR}=0 \mathrm{~dB}$ in at least three PRFs from the total of eight. A detectability map is a useful means of characterising the performance of a schedule in clutter.

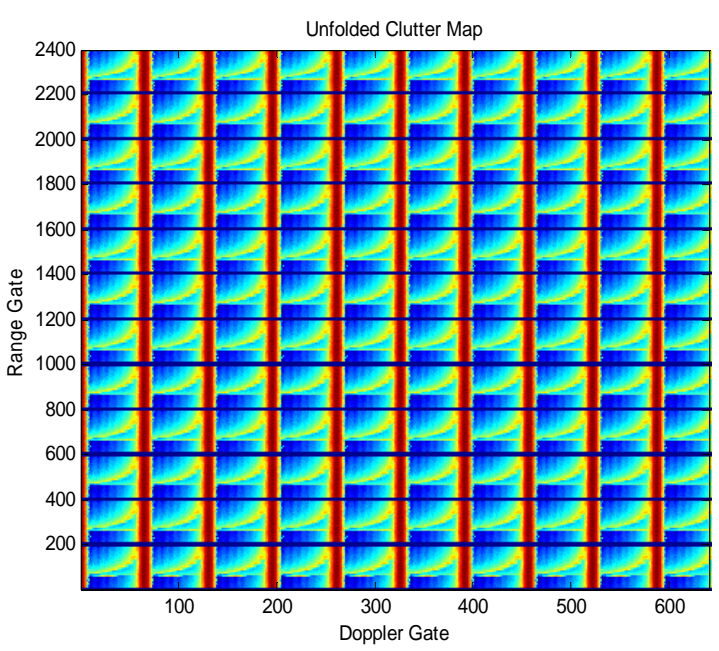

Figure 3: Unfolded Clutter Map

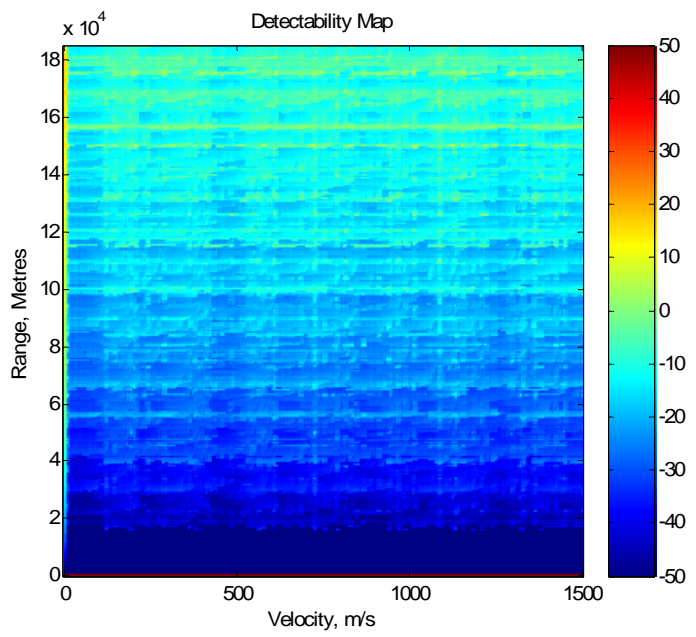

Figure 4: Detectability Map

\section{Experimental Work}

\subsection{Evolutionary Optimisation}

Figure 1 illustrates the optimisation process that has been employed in the selection of PRFs. The optimisation process is driven by an evolutionary algorithm (EA) with an optimisation goal of achieving maximum range/Doppler detectability. The EA maintains a population of trial PRF schedules whose values are refined on each iteration of the loop process (generation) along the lines of Darwinian theories of evolution and survival of the fittest. Each trial set is passed to the radar model and the genetic description is decoded to PRF values. This decoding stage employs a variety of checks to ensure that the schedule is decodable, does not incur any blind velocities, enforces a margin to minimise the risk of ghosting and is within the limits of maximum, minimum and mean PRF, as dictated by the radar model. The PRFs are passed to the radar and clutter models. The clutter model returns the clutter map for each PRF which is also passed to the radar model. The radar model is based on an airborne FCR and accepts the trial PRF schedule and clutter maps. The model then generates a detectability map. The detectability maps arising from each parent trial schedule and its offspring schedule are compared pixel by pixel (i.e. range/Doppler cells). The solution having the lowest RCS requirement in the greater number of pixels populates the next generation of trial schedules. Over repeated generations of the process, PRF schedules of superior detectability performance evolve. In this way, no detectability map is quantified for an absolute level of performance but merely compared with those of previous generations. This is known as binary tournament selection.

Evolutionary algorithms are powerful optimisation techniques which have been successfully employed in a variety of combinatorial problems. They are particularly adept at finding near-optimum solutions very quickly when the number of possible combinations precludes an exhaustive search. It is worth noting that any EA will converge to a solution, however, the efficiency of its convergence and the quality of the solution depend upon the tuning of the algorithm (e.g. population size, cross-over and mutation rates). There exists, as yet, no mathematical description of the performance of an EA. Furthermore, since the number of possibilities in the current problem is so vast, an exhaustive search of all such possibilities is not possible and there can be no confirmation that any particular solution is indeed the global optimum. Hence solutions identified in this work are termed near-optimum.

\subsection{Trials}

The optimisation has been carried out initially for a homogenous clutter scene corresponding to land clutter. 
Near-optimum PRF schedules have been identified and characterised in terms of their detectability maps. These schedules have subsequently been trialled and characterised when tested against a homogenous sea clutter scene. Similarly, PRF schedules optimised for sea clutter have been trialled against land clutter.

One might reasonably expect some degradation in detectability for those schedules optimised for one particular clutter environment (land or sea) but then used in the other clutter environment. These differences may be determined by forming the difference between the two detectability maps and applying standard logical and/or statistical measures.

Further trials have also been conducted in which the aircraft is flown above a straight coastline and the antenna mainbeam is directed ahead and slightly depressed. In this scenario the radar is subject to land clutter to one side of its track and sea clutter on the opposite side of its track; this symmetry ensures equal contributions from both sea and land clutter at all Dopplers and ranges. The PRF optimisation in these circumstances now yields values designed to meet these varied conditions.

The EA has been run 100 times in each of the clutter scenes i.e. land, sea and the mixed land/sea. This yields 100 nearoptimum solutions for each clutter scene. PRFs optimised in one clutter scene have subsequently been trialled in the alternative clutter scenes. Comparisons between the detectability of the various PRFs and clutter scenes are described in Table 2.

\begin{tabular}{|c|c|}
\hline $\begin{array}{c}\text { Clutter environment of } \\
\text { test }\end{array}$ & $\begin{array}{c}\text { PRF schedules optimised } \\
\text { environment }\end{array}$ \\
\hline Land & Land vs. Sea \\
\hline Land & Land vs. Land/Sea \\
\hline Land/Sea & Land/Sea vs. Land \\
\hline Land/Sea & Land/Sea vs. Sea \\
\hline Sea & Sea vs. Land \\
\hline Sea & Sea vs. Land/Sea \\
\hline
\end{tabular}

Table 2: Test Matrix

The first row of Table 2 above indicates that PRF schedules optimised in the land clutter environment and those optimised in the sea clutter environment are compared when their detectability maps are generated based on the presence of land clutter. The merit of each of the PRF sets optimised for the land clutter scenario is quantified as the number of its detectability maps which are superior to those of the sea optimised set. If there is real merit in using PRF sets optimised in the land clutter over those optimised for the sea clutter when tested against a land clutter scene, then the figure of merit for the land optimised PRF sets should strongly outweigh that of the sea optimised set (e.g. 90/10) at least to some level of statistical significance. If, on the other hand, there is no merit then both sets should score equally (i.e. 50/50), plus or minus a margin of statistical variation. A Wilcoxon Rank-sum test is used to assess the significance of land vs. sea optimised sets. The Wilcoxon Rank-sum test returns a test statistic which is compared to a standard normal distribution to assess the significance of the comparison. A two-tailed test is performed and at a significance of $5 \%$. Likewise, the comparisons are also made in accordance with the other rows of Table 2.

\section{Results \& Discussion}

The comparative tests defined by the test matrix of Table 2 have been conducted and the percentage significance for each test combination has been derived. Strictly, this percentage significance is the probability of being in error if one rejects the null hypothesis i.e. that the two sets of data are drawn from the same clutter distribution. These results are given in Table 3 below.

\begin{tabular}{|c|c|c|}
\hline $\begin{array}{c}\text { Clutter } \\
\text { environment } \\
\text { of test }\end{array}$ & $\begin{array}{c}\text { PRF schedules } \\
\text { optimised } \\
\text { environment }\end{array}$ & $\begin{array}{c}\text { Percentage } \\
\text { significance }\end{array}$ \\
\hline Land & Land vs. Sea & -1.67 \\
\hline Land & $\begin{array}{c}\text { Land vs. } \\
\text { Land/Sea }\end{array}$ & 21.63 \\
\hline Land/Sea & $\begin{array}{c}\text { Land/Sea vs. } \\
\text { Land }\end{array}$ & 8.56 \\
\hline Land/Sea & Land/Sea vs. Sea & -64.77 \\
\hline Sea & Sea vs. Land & 0.02 \\
\hline Sea & Sea vs. Land/Sea & 2.68 \\
\hline
\end{tabular}

Table 3: Test Statistics

A low positive percentage significance indicates that there is strong statistical evidence to support the premise that PRF sets optimised on the basis of one clutter scene (i.e. the first named scene in the middle column of Table 3) outperform PRF sets optimised for a differing clutter scene (i.e. the second named clutter scene in the middle column of Table 3 ) when tested against the former clutter scene (i.e. the scene named in the left column of Table 3). An upper limit of 5\% is commonly accepted as corresponding to a strong 
statistical correlation. Negative percentage significances disprove the premise by demonstrating the opposite. The only instance of this to any level of statistical significance is given in the first row of results in Table 3. This indicates that PRF sets optimised for sea clutter outperform those optimised for land clutter even when tested in a land clutter scene. Three sets of results (the second, third and fourth row of results in Table 3) do not exceed the threshold of statistical significance. These tests demonstrate that there is no value for optimising PRF sets for specific clutter statistics as both sets of solutions perform equally.

Whilst it may seem that there is real value in optimising PRF schedules specific to sea clutter, the authors believe that this result is due to the formation of the detectability map which favours statistical clutter distributions having an extended tail (i.e. the sea clutter). With a long tailed distribution, there are many large clutter values, but also proportionally many small values, too. As only three from eight detections are required, instances of large clutter values in one or two PRFs can be tolerated since there are likely to be three or more PRFs having very small clutter values. These smaller values will therefore dominate the detectability maps. Thus in the SLC regions the returns are reduced making the edges of the $\mathrm{MBC}$ region clearer. It appears that clear visibility of $\mathrm{MBC}$, altitude line and eclipsing are the main factors influencing the EAs ability to find optimal solutions.

\section{Conclusions}

The optimisation process has been successful in identifying several near-optimum PRF schedules for each clutter scene. The precise PRF values are valid for the FCR model parameters considered here and so are of limited general interest. Nevertheless, a comparison between the different detectability maps is valid for a wide range of radars since the trends in changes of detectability result from differing clutter scenes only. Schedules optimised for one homogenous clutter scene (land or sea) do not perform as well in the presence of clutter from the opposite environment. However, the margin of superiority is relatively small. Thus there is little value to be gained for performing the optimisation in the appropriate clutter scene. The detectability of schedules optimised for the inhomogeneous land/sea scene indicate that it is possible to design medium PRF schedules which are well suited to operations over land and sea. These schedules offer a detectability performance which is only marginally degraded with respect to those optimised for either clutter type. In summary, whilst medium PRF waveform diversity is advantageous to meet diverse operational conditions (e.g., change of altitude/velocity), it affords no real advantage in target detectability with diverse clutter scenes.

\section{References}

[1] Clive M. Alabaster, Evan J. Hughes and John H. Matthew, "Medium PRF radar PRF selection using evolutionary algorithms", IEEE Transactions on Aerospace and Electronic Systems, Vol. 39, Iss. 3, pp 990 - 1001, (July 2003).

[2] C. M. Alabaster, E. J. Hughes, S. M. Parry, D. A. Wiley, J. H. Matthew and P. G. Davies, "Medium PRF Schedules for Airborne Fire Control Radar", Proc. Waveform Diversity and Design, Edinburgh, UK, $\left(8^{\text {th }}-\right.$ $10^{\text {th }}$ November 2004).

[3] P. G. Davies and E. J. Hughes, "Medium PRF set selection using evolutionary algorithms", IEEE Transactions on Aerospace and Electronic Systems, vol. 38, no. 3, pp.933-939, (July 2002).

[4] Evan J. Hughes and Clive M. Alabaster, "Novel PRF Schedules for Medium PRF Radar", Proc. Radar 2003 Conference, Adelaide, S. Australia, $\left(3-5^{\text {th }}\right.$ September 2003). 